\title{
Is the adapted Household Food Insecurity Access Scale (HFIAS) developed internationally to measure food insecurity valid in urban and rural households of Ethiopia?
}

Seifu Hagos Gebreyesus ${ }^{1,2^{*}}$, Torleif Lunde², Damen H Mariam', Tasew Woldehanna ${ }^{3}$ and Bernt Lindtjørn²

\begin{abstract}
Background: The concept of food insecurity encompasses three dimensions. One of these dimensions, the access component of household food insecurity is measured through the use of the Household Food Insecurity Access Scale (HFIAS). Despite its application in Ethiopia and other similar developing countries, its performance is still poorly explored. Our study aims to evaluate the validity of the HFIAS in Ethiopia.

Methods: We conducted repeated cross-sectional studies in urban and rural villages of the Butajera District in southern Ethiopia. The validation was conducted on a pooled sample of 1,516 households, which were selected using a simple random sampling method. The HFIAS was translated into the local Amharic language and tested for face validity. We also evaluated the tool's internal consistency using Cronbach's alpha and factor analysis. We tested for parallelism on HFIAS item response curves across wealth status and further evaluated the presence of a dose-response relationship between the food insecurity level and the consumption of food items, as well as between household wealth status and food insecurity. Additionally, we evaluated the reproducibility of the tool through the first and second round of HFIAS scores.

Results: The HFIAS exhibited a good internal consistency (Cronbach's alpha for the values of rounds 1 and 2 were 0.76 and 0.73 , respectively). A factor analysis (varimax rotation) resulted in two main factors: the first factor described a level of mild to moderate food insecurity, while the second factor described severe food insecurity. HFIAS item response curves were parallel across wealth status in the sample households, with a dose-response trend between food insecurity levels and the likelihood of previous day food consumption being observed. The overall HFIAS score did not change over the two rounds of data collection.
\end{abstract}

Conclusions: The HFIAS is a simple and valid tool to measure the access component of household food insecurity. However, we recommend the adaptation of questions and wordings and adding examples before application, as we found a discrepancy in understanding of some of the nine HFIAS questions.

Keywords: HFIAS, Food security, Validity

\footnotetext{
* Correspondence: Seif_h23@yahoo.com

${ }^{1}$ Department of Reproductive Health and Health Service Management,

School of Public Health, College of Health Sciences, Addis Ababa University,

Addis Ababa, Ethiopia

${ }^{2}$ Center for International Health, University of Bergen, Bergen, Norway

Full list of author information is available at the end of the article
} 


\section{Background}

In its Plan of Action, the 1996 World Food Summit adopted a working definition on food security. This definition was redefined in 2001 by the Food and Agricultural Organization (FAO): "Food security [is] a situation that exists when all people, at all times, have physical, social and economic access to sufficient, safe and nutritious food that meets their dietary needs and food preferences for an active and healthy life" [1]. According to a recent estimate by the FAO, approximately 870 million people worldwide are undernourished, with $27 \%$ of these affected people residing in sub-Saharan African countries [2].

Food security is a complex issue with a multi-dimensional concept, which is based on multiple dimensions such as physical, social, and economic access, availability, amount, preferences for certain foods, security, and time [3]. The measurement of food insecurity at any given time captures one if not more of the three dimensions of food security: availability, utilization, and access. The food insecurity assessment based on the availability dimension is widely used and for the most part guides the responses to food insecurity [4]. However, it fails to capture the unequal distribution of food and is also unable to guarantee the utilization of food in a given population. An assessment of food insecurity based on the second dimension, utilization, is well captured through various anthropometric indicators, e.g., underweight, stunting, and wasting. Nonetheless, measurements based on the access dimension of food insecurity are not yet well established.

Food access, which reflects the demand side of food security, has recently been designated as one of the major contributors to food insecurity [5]. In 2006, the USAID-funded Food and Nutrition Technical Assistance Project, through the Academy for Educational Development, published a tool that measures the access component of household food insecurity. The tool was developed to be simple, easy to use, and applicable, with only minor adaptations to different sociocultural contexts. The tool captures three domains: i) anxiety and uncertainty about food access, ii) insufficient quality (variety, preferences, and social acceptability), and iii) insufficient food intake and the physical consequences [6].

The HFIAS has been shown to measure food insecurity with an acceptable standard in a few developing countries [7-10]. Even so, a lot has been done on measurements of the access component of food insecurity in developed countries such as the US [11]. The Core Food Security Module (CFSM), which has a similar structure with that of the HFIAS, is currently being used to measure the access component of food insecurity in the US. The CFSM is based on a set of 18 questions for households with children and 10 questions for households without children, and the frequencies of affirmative responses to these questions are used to discriminate households into three levels of food insecurity [12].

Maes and colleagues have attempted to validate the HFIAS among volunteer AIDS caregivers in Addis Ababa, Ethiopia [9]. The authors reported that the tool performed well in capturing the access component of food insecurity among the study participants, who were special groups of people and may not represent the general population.

However, despite the increasing application of the tool in Ethiopia, its performance remains underexplored. The results of this study will help to strengthen the applicability of the tool and its performance for measuring progress and to monitor and evaluate different programs focusing on household food insecurity.

\section{Methods}

\section{Study setting}

The study was conducted in the Butajera District of southern Ethiopia, which is located approximately $130 \mathrm{~km}$ from Addis Ababa (the capital city of Ethiopia) in the Guraghe Zone in the Southern Nations Nationalities and People's Region (SNNPR). The district houses a Rural Health Program (BRHP) (owned and operated by Addis Ababa University), which is a health and demographic surveillance system (HDSS) with a continuous registration of vital and migratory events among ten selected villages. The studied district was purposely selected for the benefit of a better sampling frame and research infrastructure.

\section{Study design and period}

A community-based, cross-sectional study design was employed between November and December 2013. We administered the survey questionnaire twice to the study participants, and the second survey questionnaire was administered to the study participants after 7 days of the first administration. This repeated survey was used to determine the reproducibility of the household food insecurity assessment tool (HFIAS).

\section{Study population and sampling}

The study included a total of ten HDSS villages, of which nine were rural and one was urban, and the study population included households residing in these villages.

The sample size for the study was estimated using the formula for a single population proportion. Assuming an $80 \%$ prevalence of household food insecurity [13], a $95 \%$ confidence level, a $4 \%$ margin of error, and a design effect of 2 , the calculated sample size was 768 households. With an expectation of a $5 \%$ non-response rate, the final sample size required was approximately 800 households.

The final sample size was allocated to the ten HDSS villages proportionate to the number of households in each village. We then used BRHP data set as a sampling 
frame and applied a simple random sampling method to select study households within a given village.

\section{Data collection}

The HFIAS is composed of nine items, which are asked with a recall period of 1 month. For each item, there was a follow-up of the frequency of the occurrence question. The tool was also translated into the Amharic (local) language by one of the authors (SHG) and initially reviewed with research assistants who were residents in the study area.

\section{Face validity}

We discussed all nine questions independently with four urban and four rural households in the neighboring villages, basically aiming at whether the questions were clear, easily understandable, and had a minimal amount of multiple interpretations. We read the nine questions to the women, and the responses were recorded. This was followed by a question about how the women understood each question. For example, we asked: "What do you understand when I ask you the question: In the past four weeks, were you or any household member not able to eat the kinds of foods you preferred because of a lack of resources?" We compared their understanding with that of the primary aim of the questions, and when there was a difference between what they understood and what we were actually looking for, a discussion followed on how that question could best be framed to make it clearer and contextually appropriate. Lastly, together with these women, the nine questions were adapted through modification, rephrasing, and adding examples when necessary.

We collected food groups that a household had consumed over the preceding $24 \mathrm{~h}$ [14], with the household food intake structured using the consumption of $12 \mathrm{food}$ groups/item. The food groups included meat, fish, vegetables, fruits, eggs, potatoes, and other roots/tubers, beans, cereals/breads, oil, fat or butter, sugar or honey, as well as other types of foods such as coffee and tea.

Moreover, a range of sociodemographic data about the respondents such as age, education, religion, marital status, and occupation was collected, in addition to household-level data such as ownership and size of land, type of house and construction materials, availability of fixed assets such as radio, television, phone, bed and chair, and other household items, possession of domestic animals, type of water source for drinking and cooking, and availability and type of latrine.

Interviews were conducted by 20 trained and experienced junior nurses who are residents of the local district and had similar data collection experiences. The work was monitored by six supervisors, and interviews were primarily conducted with women in the household, as women are commonly responsible for food preparation in the study area. If women were unavailable, another adult who was present and ate in the household the previous day was asked.

\section{Quality control}

Questionnaires were controlled for completeness and logical errors, and where errors were found, the questionnaires were redone. Consistency checks were done to improve the quality of the data, and inconsistent entries and responses were crosschecked with the questionnaires and corrected accordingly.

\section{Ethical clearance}

The study protocol was approved by institutional review boards from the Addis Ababa University, College of Health Sciences. The study was also approved by the Regional Committee for Medical and Research Ethics, Western Norway (REK Vest). Information on the research objective was read to the participants, verbal informed consent was received, and the privacy and confidentiality of respondents was also maintained.

\section{Data entry and analysis}

We used EpiData Version 3.1 for the data entry, and the data was exported to Stata 11.0 (StataCorp, College Station, TX) for cleaning and further analysis.

Household wealth was constructed through a principal component analysis (PCA) of the household-level data described above. The PCA was done independently for urban and rural samples, and the score was then used to assign sampled households into quintiles that indicate poorest, poor, medium, rich, and richest.

The results from HFIAS delineate households across the four levels of food insecurity, including food secure, mild food insecure, moderate food insecure, and severely food insecure. The procedure and steps used to assign households to one of the levels is described elsewhere [3].

\section{Factor analysis}

An exploratory analysis was conducted on the nine items, using a Horn's parallel analysis (PA) to determine the number of factors to retain. PA is a Monte Carlobased simulation method that compares observed eigenvalues with that obtained from uncorrelated normal variables.

\section{Validation}

We evaluated the validity of the nine-item food insecurity assessment tool based on the following recommended criteria employed by a few similar studies $[9,15,16]$.

The first criterion is the value of the Cronbach's alpha, which is a measure for internal consistency, approaching 0.85 for the two rounds of surveys. Secondly, we tested for parallelism on HFIAS item response curves across 
Table 1 Sample characteristics of households by rounds of data collection, Ethiopia 2013

\begin{tabular}{|c|c|c|}
\hline Variable & Round I (\%) & Round II (\%) \\
\hline$N$ & 767 & 749 \\
\hline \multicolumn{3}{|l|}{ Residency, \% } \\
\hline Urban & 30.4 & 30.3 \\
\hline Rural & 69.6 & 69.7 \\
\hline \multicolumn{3}{|l|}{ Respondent status, \% } \\
\hline Household head & 13.3 & 12.7 \\
\hline Spouse & 62.1 & 56.1 \\
\hline Other adult male & 5.8 & 5.6 \\
\hline Other adult female & 18.8 & 25.6 \\
\hline \multicolumn{3}{|l|}{ Missing } \\
\hline \multicolumn{3}{|l|}{ Reported age, \% } \\
\hline $14-29$ years & 33.0 & - \\
\hline $30-45$ years & 45.2 & - \\
\hline $46-61$ years & 14.2 & - \\
\hline $61+$ years & 7.6 & - \\
\hline Mean age & 36.9 & \\
\hline \multicolumn{3}{|l|}{ Marital status, $\%$} \\
\hline Single & 15.3 & - \\
\hline Married & 65.1 & - \\
\hline Separated & 1.7 & - \\
\hline Divorced & 2.0 & - \\
\hline Widowed & 14.7 & - \\
\hline Missing & 1.3 & - \\
\hline \multicolumn{3}{|l|}{ Religion } \\
\hline Orthodox Christian & 18.6 & - \\
\hline Muslim & 73.7 & - \\
\hline Protestant & 7.2 & - \\
\hline Catholic & 0.5 & - \\
\hline Missing & 0.4 & - \\
\hline \multicolumn{3}{|l|}{ Occupation } \\
\hline Housewife & 32.5 & - \\
\hline Farmer and housewife & 31.3 & - \\
\hline Merchant & 14.3 & - \\
\hline Students & 9.0 & - \\
\hline Daily laborer & 4.3 & - \\
\hline Employee & 4.2 & - \\
\hline Others & 4.4 & - \\
\hline \multicolumn{3}{|l|}{ Fl level, \% } \\
\hline Food secure & 11.9 & 9.6 \\
\hline Mild FI & 21.6 & 23.1 \\
\hline Moderate FI & 50.3 & 58.1 \\
\hline Severe FI & 16.2 & 9.2 \\
\hline
\end{tabular}

Table 1 Sample characteristics of households by rounds of data collection, Ethiopia 2013 (Continued)

\begin{tabular}{lll}
\hline HFIAS score & $6.1 \pm 4.5^{\mathrm{a}}$ & $6.3 \pm 4.2$ \\
Dietary diversity score (0-12) & $5.2 \pm 1.5^{\mathrm{a}}$ & $5.2 \pm 1.6$ \\
\hline${ }^{\mathrm{a}}$ Mean and standard deviations. &
\end{tabular}

wealth status, which was done by comparing the likelihood of affirmative responses to the nine items across households' wealth quintiles.

Thirdly, we evaluated the presence of a dose-response relationship between food insecurity level and the previous day consumption of certain food items. We also tested for a dose-response relationship between household wealth status and food insecurity levels and used the extended Mantel-Haenszel chi square for linear trend to check for dose-response relationships.

Additionally, the reproducibility between the first and second HFIAS scores (HFIAS overtime) was estimated by means of a paired $t$-test.

\section{Results}

Sample characteristics

A total of 1,516 households (767 in the first round and 749 in the second round) were studied across the two rounds of data collection. The response rate for the first and second round of data collection was $96 \%$ and $98 \%$, respectively, and we included 1,056 and 460 households from rural and urban villages, respectively.

The sample characteristic of the study population is shown in Table 1, and the mean age (year) of the respondents was 36.9 years. The age distribution indicated that $33 \%$ and $45.2 \%$ of respondents were in the age group between 14-29 and 30-45, respectively, and the great majority were Muslims (73.7\%), rural residents (69.6\%), and married (65.1\%). Occupationally, 32.5\% were housewives and $31.3 \%$ were a combination of a housewife/farm worker.

\section{Responses to the nine HFIAS items}

As seen in Table 2, affirmative responses for the items ranged from $2.0 \%$ to $76.1 \%$ and $0.1 \%$ to $80.3 \%$ among urban and rural samples, respectively. We found that affirmative responses were highest for items showing mild to moderate forms of food insecurity such as worry about food, unable to eat preferred foods, eating a limited variety of food items, and eating smaller or fewer meals a day. Among urban samples, the item that received the highest affirmative response was item 3: “...Did you or any household member have to eat a limited variety of foods?" For the rural samples, item 2:"Were you or any household member not able to eat the kinds of foods you preferred?" received the highest affirmative response. Affirmative responses for items 7, 8, and 9, which indicates severe forms of food insecurity, were low. Of the nine items, the 
Table 2 Affirmative responses to items on the Household Food Insecurity Access Scale (HFIAS) in urban and rural settings, Ethiopia, 2013

\begin{tabular}{|c|c|c|c|c|c|c|}
\hline \multirow[t]{2}{*}{ HFIAS questions } & \multicolumn{2}{|c|}{ Urban } & \multicolumn{2}{|c|}{ Rural } & \multicolumn{2}{|c|}{ Total } \\
\hline & $n$ & (\% yes) & $n$ & (\% yes) & $n$ & (\% yes) \\
\hline Q1. Worry about food & 308 & 66.96 & 549 & 51.99 & 857 & 56.5 \\
\hline Q2. Unable to eat preferred foods & 355 & 71.17 & 848 & 80.3 & 1203 & 79.35 \\
\hline Q3. Eat a limited variety of foods & 350 & 76.09 & 728 & 68.94 & 1078 & 71.11 \\
\hline Q4. Eat foods that you really did not want to eat & 9 & 1.96 & 7 & 0.09 & 10 & 0.66 \\
\hline Q5. Eat a smaller meal & 284 & 61.74 & 540 & 51.14 & 824 & 54.35 \\
\hline Q6. Eat fewer meals in a day & 277 & 60.22 & 586 & 55.49 & 863 & 56.93 \\
\hline Q7. No food to eat of any kind in the household & 40 & 8.7 & 39 & 3.69 & 79 & 5.21 \\
\hline Q8. Go to sleep at night hungry & 75 & 16.3 & 74 & 7.01 & 149 & 9.83 \\
\hline Q9. Go a whole day and night without eating anything & 27 & 5.87 & 28 & 2.65 & 55 & 3.63 \\
\hline
\end{tabular}

item: "Eat food that I really did not want to eat" received the lowest affirmative responses in both the urban and rural samples.

\section{Face validity}

We conducted a total of eight interviews (four each among rural and urban households), with the main aim of these interviews being to get the wording right for the specific context of the use of the HFIAS. We found that four of the nine questions were not straightforward and needed to be modified in either way for a better understanding. We discussed with these nine women how to best frame these questions to help make them clearer and contextually appropriate without losing the main aim of these questions. The questions that needed modifications, rephrasing, or adding examples were items $2-4$ and item 6 (Table 2). The remaining five questions were kept in their original form since they were not difficult to understand and did not have multiple interpretations.

According to these interviews, we found a higher likelihood of affirmative response to the item "unable to eat preferred food." This item was restructured, and examples were necessary to reflect a preferred food according to the respondent's own economy, rather than a general preference. Interviewees better understood the item "... limited variety of foods" if the item was translated with a core meaning of "monotonous diet" or "almost the same meal every time." The item "...eat food that you really don't want to eat" was also rephrased as food items that are not eaten under normal circumstances, but could be eaten during times of hardship, such as during a severe food crisis or a severe drought. Two to three meals per day were considered as a normal meal frequency in the study district. Hence, the item "...eat fewer meals a day" was modified as "...eat less than two-three meals per day." In summary, the key adaptations we made included rephrasing, adding local (context-specific) examples, as well as specifying the name of the previous month. Furthermore, the importance of explaining the objective of the study in detail prior to initiating the interview process was found to be vital in obtaining a correct response.

\section{Exploratory analysis}

A factor analysis (varimax rotation) of the nine HFIAS questions resulted in two main factors for both urban and rural samples (in fact, the analysis resulted in nine factors, though the PA indicated to retain two factors). The first factor loaded most highly on the first five HFIAS items (except for item 4), while the second factor loaded most highly on the last three items (Table 3 ). The first factor seems to describe the level of a mild to moderate form of food security, while the second factor seems to describe the level of a severe form of food insecurity. Factors 1 and 2 together explained 56.7\% and $54.3 \%$ of the nine questions' combined variance for rural and urban samples, respectively.

\section{Internal consistency}

The internal consistency analysis showed that the Cronbach's alpha values for the overall sample (both rural and urban samples) for rounds 1 and 2 were 0.73 and 0.73 , respectively (Table 4 ), whereas the Cronbach's alpha values were relatively higher among urban residents compared to those among rural residents in the two rounds of data collection.

\section{Parallelism}

Figures 1 and 2 show HFIAS item response curves across household wealth status in the urban and rural villages of the study district. HFIAS item response curves were parallel across wealth status in urban households, and with the exception of HFIAS item 4, we observed that the likelihood of affirmative responses decreased as the household wealth status increased. When compared to 
Table 3 Factor loadings for rotated component matrix for households' responses to nine questions by residency, Ethiopia, 2013

\begin{tabular}{|c|c|c|c|c|c|c|}
\hline \multirow[t]{3}{*}{ HFIAS questions } & \multicolumn{6}{|c|}{ Factor loading } \\
\hline & \multicolumn{2}{|l|}{ Rural } & \multicolumn{2}{|l|}{ Urban } & \multicolumn{2}{|l|}{ Total } \\
\hline & Factor 1 & Factor 2 & Factor 1 & Factor 2 & Factor 1 & Factor 2 \\
\hline Q1. Worry about food & 0.6861 & 0.1336 & 0.7042 & 0.0951 & 0.6916 & 0.1384 \\
\hline Q2. Unable to eat preferred foods & 0.6817 & -0.0504 & 0.7833 & 0.0852 & 0.7089 & -0.0079 \\
\hline Q3. Eat a limited variety of foods & 0.5618 & 0.1296 & 0.6832 & 0.0486 & 0.6061 & 0.1084 \\
\hline Q4. Eat foods that you really did not want to eat ${ }^{a}$ & - & - & 0.2427 & -0.0984 & 0.1664 & -0.0186 \\
\hline Q5. Eat a smaller meal & 0.7874 & 0.1954 & 0.7782 & 0.2547 & 0.7846 & 0.2166 \\
\hline Q6. Eat fewer meals in a day & 0.7578 & 0.1978 & 0.8157 & 0.1708 & 0.7769 & 0.1853 \\
\hline Q7. No food to eat of any kind in the household & 0.0766 & 0.7384 & 0.1245 & 0.7295 & 0.0973 & 0.7416 \\
\hline Q8. Go to sleep at night hungry & 0.2104 & 0.8220 & 0.2346 & 0.8188 & 0.2285 & 0.8184 \\
\hline Q9. Go a whole day and night without eating anything & 0.0616 & 0.8352 & 0.0506 & 0.7623 & 0.0584 & 0.8065 \\
\hline
\end{tabular}

Extraction method: principal component analysis, rotation method: Varimax. Kaiser-Meyer-Olkin measure of sampling adequacy $=0.80$.

anwanted food dropped in because of zero variance.

the urban sample, a similar but less clear pattern is observed for rural households.

\section{Food insecurity level and food intake}

Among rural sample households, a dose-response trend between the food insecurity level and the likelihood of the previous day's consumption of food items such as eggs, milk, fish, cereals, and beans was observed. For example, the likelihood of the previous day's consumption of milk among food-secure households was 39.8\% compared with $11.6 \%$ for severely food-insecure households. Similarly, the likelihood of the previous day's consumption of eggs among food-secure households was $10.2 \%$ as compared with $2.1 \%$ for severely food-insecure households. The observed trend between food-secure and foodinsecure households was significant for eggs $(p<0.018)$, milk $(p<0.01)$, fish $(p<0.01)$, cereals $(p<0.01)$, and pulses $(p<0.01)$, with the result also indicating that some food items such as vegetables and roots were less sensitive to household food insecurity levels. These food items are common staples in the diet for the rural parts of the studied district.

Among urban sample households, with the exception of the consumption of fish, dose-response trends between food insecurity level and the likelihood of the previous day's consumption of other food items were observed. Additionally, the observed trends between food-secure

Table 4 Internal consistency (Cronbach's alpha) by rounds of data collection and residency, Ethiopia 2013

\begin{tabular}{lll}
\hline Residency & Rounds & \\
\cline { 2 - 3 } & $\mathbf{I}$ & $\mathbf{~ I I}$ \\
\hline Urban & 0.79 & 0.80 \\
Rural & 0.75 & 0.68 \\
Total & 0.76 & 0.73 \\
\hline
\end{tabular}

and food-insecure households were also significant for all the food items that showed a dose-response trend.

\section{FI severity and household wealth status}

We found a significant and positive dose-response trend $(p<0.01)$ between household wealth status and levels of food security among rural and urban samples. With the exceptions of minor deviations between the two lowest (poorest and poor) strata, an increase in household wealth has been accompanied by an improved household food security.

An inverse but significant dose-response trend between $(p<0.01)$ household food insecurity level and wealth status was found among the urban samples (Figure 3). In the urban samples, a decrease in household wealth is accompanied by a higher household food insecurity level, though we did not find a significant dose-response trend between the household wealth and food insecurity level among rural households.

\section{FI over time (reproducibility)}

The overall HFIAS score did not change over the two rounds of data collection. The HFIAS scores for the first and second round of data collection were $6.1 \pm 4.5$ and $6.3 \pm 4.2$, respectively (Table 1 ), while the HFIAS score for the urban sample did not show a statistically significant difference across the two rounds of data collection. However, we found an increase in the HFIAS score for rural samples during the second round of data collection, and the difference in HFIAS score between the two rounds was also statistically significant (mean difference: -0.58 ; $95 \%$ CI: $-1.07,-0.083)$.

\section{Discussion}

In this study, we validated an adapted version of the HFIAS developed to measure the access component of 


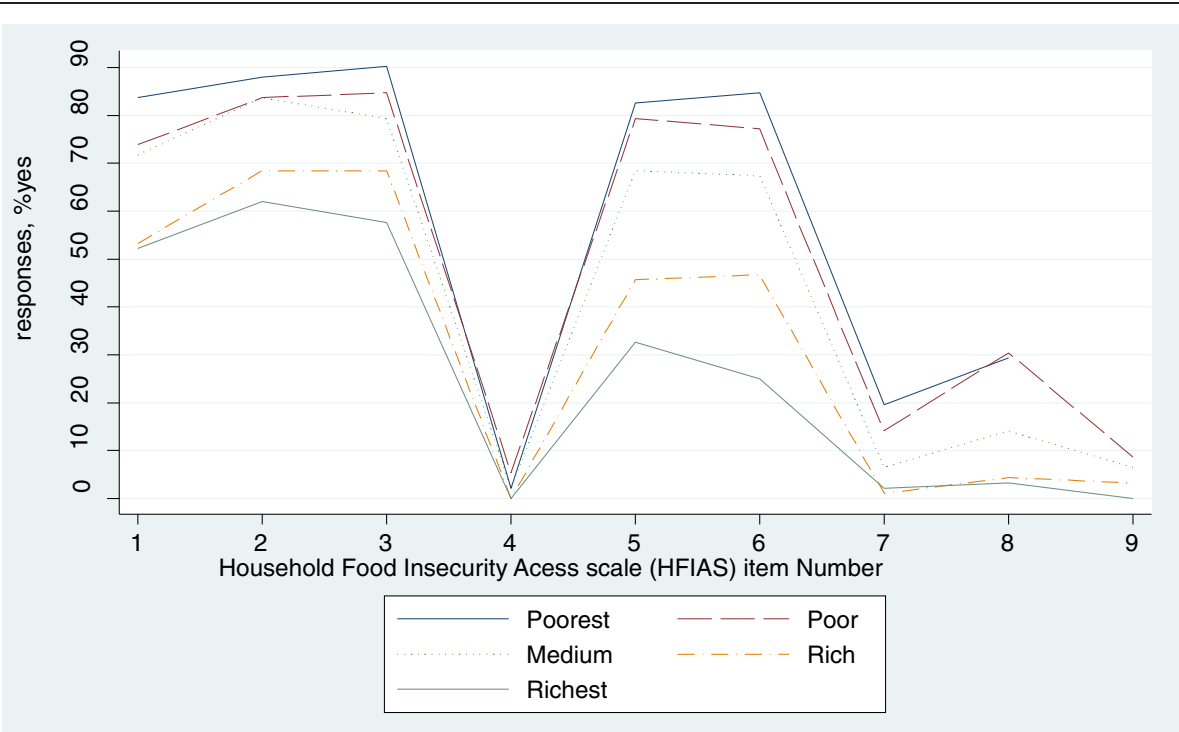

Figure $1 \mathrm{HFIAS}$ item response curves across household wealth quintile strata among 233 urban households. In the town of Butajera, Ethiopia, 2013. Observations across two data collection rounds are pooled (combined $n=460$ ).

food insecurity among both rural and urban households of the Butajera District in Ethiopia. We evaluated the tool for its internal consistency, criterion validity, and reproducibility through two rounds of data collection, and our results indicate that the tool had a satisfactory internal consistency and reproducibility and performed well with minor deviations to the set criteria. The HFIAS required minor modifications such as rephrasing words, the use of local ways of expressing the questions, and adding local examples to the nine items. Most importantly, we found that providing information during the consent process on the objectives of the administration of the instruments (surveys) helped modify the respondent's expectations and get the nearest accurate responses.

A factor analysis of the nine HFIAS items discriminated between two main components, which indicate the degrees of household food insecurity level. Similar to our study, a validation study from Iran [10] reported two components. The main difference was that in our

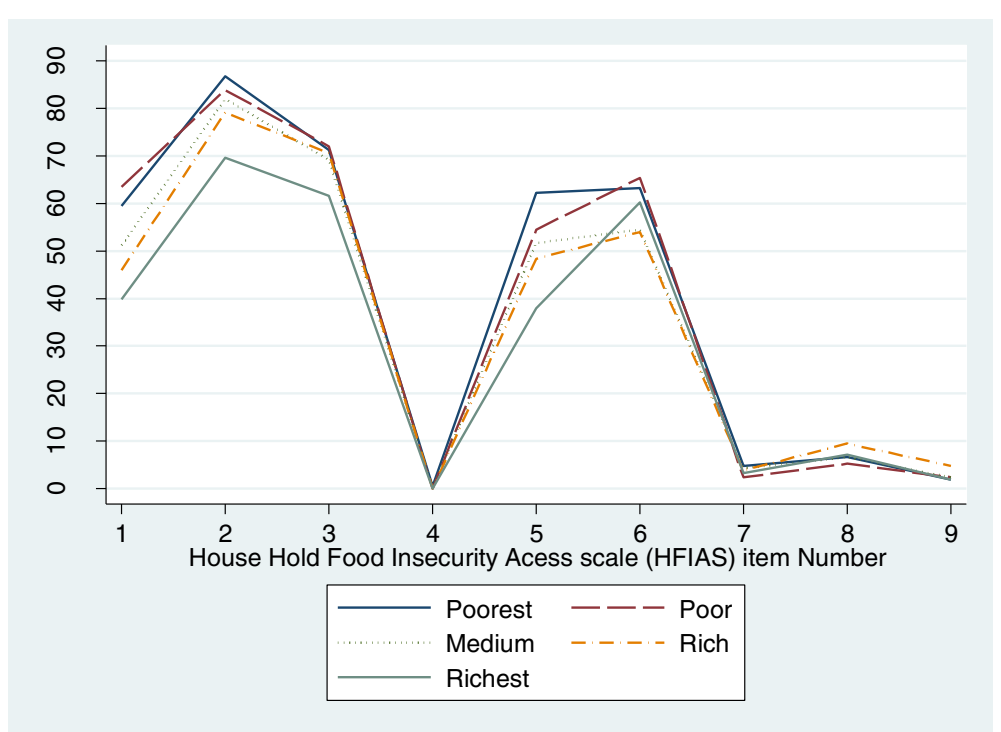

Figure 2 HFIAS item response curves across household wealth quintile strata among $\mathbf{5 3 4}$ households. In the rural villages of Butajera, Ethiopia, 2013. Observations across two data collection rounds are pooled (combined $n=1,056$ ). 


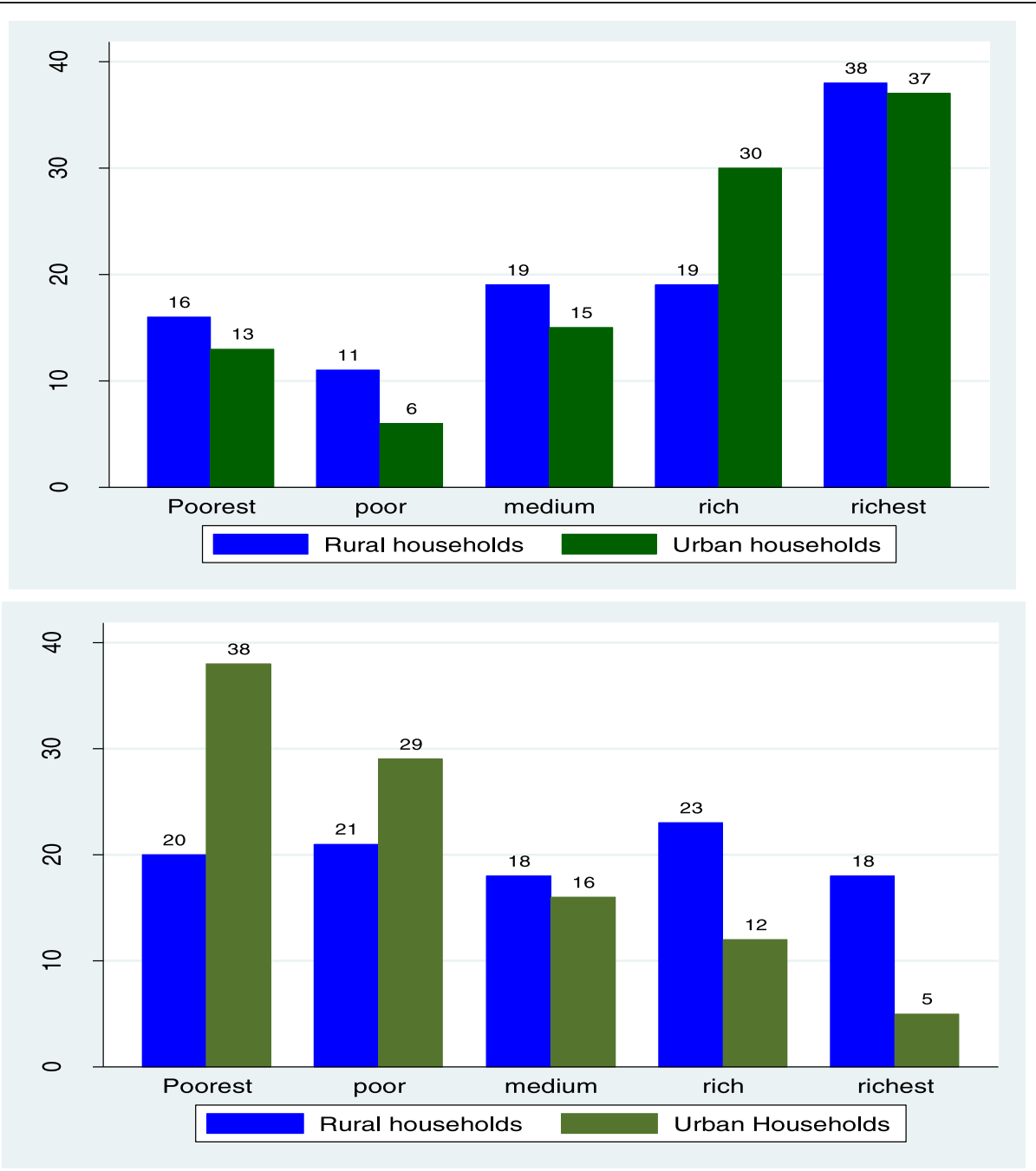

Figure 3 Food security and severe $\mathrm{FI}$ as a function of wealth quintile strata. Among 534 rural and 233 urban households in the Butajera, Ethiopia, 2013. Observations across two data collection rounds are pooled (combined $n=1,516$ ).

study, the second factor loaded on the last three items, which indicates severe forms of food insecurity, while the study from Iran reported that the second factor loaded on the last four items. Knupeel et al. [8] also reported (i) insufficient food quality and (ii) insufficient food intake as the two main factors that emerged after a rotated principal component factor analysis. Unlike the domain described by Coates et al. [3], our study showed that the first item does not appear to form a separate domain and was loaded to the first principal component. In addition to this, the fourth item does not appear to represent any specific domain.

The items that indicate a moderate food insecurity (FI) are experienced more frequently than those that indicate severe forms of FI on the HFIAS. The nine HFIAS items are sequenced in order of an increasing severity of household food insecurity [6]. In this scale, the first and ninth items are the least and most severe indicator of food insecurity, respectively. However, we found a lack of this sequential pattern on some of the nine items, e.g., for item 1: "Did you worry about having enough food?", which according to the scale should receive the highest percentage of affirmative response. Even so, the item actually only received the third highest percentage of affirmative responses. Although we cannot totally rule out the possibility of the respondent's difficulties in understanding the items, households in FI areas could adapt themselves to the existing food shortage, and "worrying" may not be the immediate response to household food shortages. Knupeel et al. [8] similarly documented a reduction in the quality and quantity of food as a first response, rather than expressing a worry about food shortage. The authors suggest that the severity of FI in these areas could alter a household's response to the item.

Another example is that the percentage of households with an affirmative response on the item "...eating fewer 
meals?" was higher than that for the item "...eating smaller meals?", which was documented among rural samples. Households in the rural areas may respond to food insecurity primarily through eating fewer meals, followed by eating smaller meals. This lack of order could indicate the contextual differences in responses to the level of FI in the households [17]. Hence, a further investigation on the order of experience of the nine items in the scale in response to the progression of household FI is warranted.

Cronbach's alpha values reported by other similar studies were relatively higher $(\alpha>0.80)$ than those by ours [8-10,16,18-22]. However, the internal consistency of HFIAS in this study is satisfactory for its application [23], and minor deviations of parallelism on the last three items were observed among the rural samples. We think that these deviations might indicate that the poor and poorest strata may have employed different coping mechanisms than the relatively rich strata. Similar deviations were also reported elsewhere [9], with the authors explaining that income strata may not necessarily translate into access to diet, as there could be less significant practical differences across the income strata.

The mean HFIAS score did not change over the two rounds of data collection for the overall sample, which shows in part that the HFIAS has a very good reproducibility to measure and capture household FI. Nevertheless, we observed a lack of reproducibility among rural samples. We do not think that changes in the HFIAS could be a real phenomenon since the interval between the administration of the first and second questionnaire was shorter (7 days), and changes related to FI are not expected. Moreover, we did not observe a significant change in household dietary diversity scores between the two rounds. Households' expectations of support after the survey and respondent's change during the second survey might be possible explanations for the lack of reproducibility among the rural samples.

\section{Limitations and strengths of the study}

Our study has some limitations that warrant consideration. The study was done in one of the nine regions of Ethiopia where the applicability of the findings may be limited to similar groups in southern Ethiopia. Respondent understanding of the nine items and expectations of possible support may have also influenced the results, and respondents could lean towards affirmative responses. Furthermore, in the absence of an established gold standard for household food insecurity, it becomes difficult to discuss the external validity of the HFIAS.

Among the main strengths of this study are the inclusion of urban and rural residents, the employment of a relatively larger sample size, and the application of a simple random sampling method to recruit households. These could help strengthen the generalizability of the findings and its application to similar contexts. We also administered two rounds of surveys in the same households to evaluate the repeatability of the HFIAS. However, this validation study was done at the household level, so further work might be needed to evaluate whether the tool can perform well if applied at the individual level (e.g., with adolescents) to help measure FI.

\section{Conclusions}

We conclude that the HFIAS is a simple and valid tool to measure the access component of household FI in urban and rural settings. However, we recommend a modification of the questions before application because we found a discrepancy in understanding on some of the nine HFIAS questions. We recommend further studies in other parts of the country, as variations in sociocultural settings could influence the successful application and validity of the tool. Moreover, we recommend that further works should also focus on identifying the correct sequence of the nine items in response to the severity of household FI. The order of progression of the nine items in the HFIAS (in the context of the level of household food insecurity) will have its own implications for the assignment of households to the different categories of food insecurity.

\section{Competing interests}

The authors declare that they have no competing interests.

\section{Authors' contributions}

SHG and BL designed the study. All authors participated in the data analysis and drafting of the manuscript. All authors read and approved the final manuscript.

\section{Acknowledgements}

We would like to acknowledge the Center for International Health at the University of Bergen for funding the study, and we are very thankful to all the study participants for their willingness to participate in the study. Lastly, we declare that the funding body had no role in the design of the study, the collection, analysis and interpretation of the data, the writing of this manuscript, and in the decision to submit it for publication.

\section{Author details}

${ }^{1}$ Department of Reproductive Health and Health Service Management, School of Public Health, College of Health Sciences, Addis Ababa University, Addis Ababa, Ethiopia. ${ }^{2}$ Center for International Health, University of Bergen, Bergen, Norway. ${ }^{3}$ Department of Economics, College of Business and Economics, Addis Ababa University, Addis Ababa, Ethiopia.

Received: 13 June 2014 Accepted: 20 November 2014 Published: 21 January 2015

\section{References}

1. Food and Agricultural Organization: The State of Food Insecurity in the World. Rome: Food and Agricultural Organization; 2002.

2. FAO, WFP, IFAD: The State of Food Insecurity in the World 2012. Economic growth is necessary but not sufficient to accelerate reduction of hunger and malnutrition. Rome: FAO; 2012.

3. Jennifer CJ, Swindale A, Bilinsky P: Household Food Insecurity Access Scale (HFIAS) for Measurement of Household Food Access: Indicator Guide (v. 3). Washington, D.C: Food and Nutrition Technical Assistance Project, Academy for Educational Development; 2007.

4. Barrett CB: Measuring food insecurity. Science 2010, 327:825-828. 
5. Sen AK: Poverty and famines: An easy on entitlement and deprivation. ; 2014.

6. Swindale A, Bilinsky P: Development of a universally applicable household food insecurity measurement tool: process, current status, and outstanding issues. J Nutr 2006, 136:1449S-1452S.

7. Becquey E, Martin-Prevel Y, Traissac P, Dembele B, Bambara A, Delpeuch F: The household food insecurity access scale and an index-member dietary diversity score contribute valid and complementary information on household food insecurity in an urban West-African setting. J Nutr 2010, 140:2233-2240.

8. Knueppel D, Demment M, Kaiser L: Validation of the Household Food Insecurity Access Scale in rural Tanzania. Public Health Nutr 2010, 13:360-367.

9. Maes KC, Hadley C, Tesfaye F, Shifferaw S, Tesfaye YA: Food insecurity among volunteer AIDS caregivers in Addis Ababa, Ethiopia was highly prevalent but buffered from the 2008 food crisis. J Nutr 2009, 139:1758-1764.

10. Mohammadi F, Omidvar N, Houshiar-Rad A, Khoshfetrat MR, Abdollahi M, Mehrabi Y: Validity of an adapted Household Food Insecurity Access Scale in urban households in Iran. Public Health Nutr 2012, 15:149-157.

11. Gundersen C: Food insecurity is an ongoing national concern. Adv Nutr 2013, 4:36-41.

12. Coleman-Jensen A, Nord M, Singh A: Household Food Security in the United States in 2012. U.S. Department of Agriculture, Economic Research Service: ERR-155; 2013.

13. Regassa N, Stoecker BJ: Contextual risk factors for maternal malnutrition in a food-insecure zone in southern Ethiopia. J Biosoc Sci 2012, 44:537-548.

14. F.A.O: Guidelines for Measuring Household and Individual Dietary Diversity. Rome, Italy: F.A.O; 2013.

15. Frongillo EA Jr: Validation of measures of food insecurity and hunger. J Nutr 1999, 129:506S-509S.

16. Perez-Escamilla R, Segall-Correa AM, Kurdian ML, Sampaio Md MF, Marin-Leon L, Panigassi G: An adapted version of the U.S. Department of Agriculture Food Insecurity module is a valid tool for assessing household food insecurity in Campinas, Brazil. J Nutr 2004, 134:1923-1928.

17. Coates J, Frongillo EA, Rogers BL, Webb P, Wilde PE, Houser R: Commonalities in the experience of household food insecurity across cultures: what are the measures missing? I Nutr 2006, 136:1438S-1448S

18. Gulliford MC, Mahabir D, Rocke B: Reliability and validity of a short form household food security scale in a Caribbean community. BMC Public Health 2004, 4:22.

19. Kendall A, Olson CM, Frongillo EA Jr: Validation of the Radimer/Cornell measures of hunger and food insecurity. J Nutr 1995, 125:2793-2801.

20. Leyna GH, Mmbaga EJ, Mnyika KS, Klepp Kl: Validation of the Radimer/ Cornell food insecurity measure in rural Kilimanjaro, Tanzania. Public Health Nutr 2008, 11:684-689.

21. Vargas S, Penny ME: Measuring food insecurity and hunger in Peru: a qualitative and quantitative analysis of an adapted version of the USDA's Food Insecurity and Hunger Module. Public Health Nutr 2010, 13:1488-1497.

22. Zerafati SN, Omidvar N, Ghazi-Tabatabaie M, Houshiar RA, Fallah H, Mehrabi $Y$ : Is the adapted Radimer/Cornell questionnaire valid to measure food insecurity of urban households in Tehran, Iran? Public Health Nutr 2007, 10:855-861.

23. Henerson ME, Morris LL, Fitz-Gibbon CT: How to Measure Attitudes. Beverly Hills, CA: Sage Publications; 1978.

doi:10.1186/2055-0928-1-2

Cite this article as: Gebreyesus et al:: Is the adapted Household Food Insecurity Access Scale (HFIAS) developed internationally to measure food insecurity valid in urban and rural households of Ethiopia? BMC Nutrition 2015 1:2.

\section{Submit your next manuscript to BioMed Central and take full advantage of:}

- Convenient online submission

- Thorough peer review

- No space constraints or color figure charges

- Immediate publication on acceptance

- Inclusion in PubMed, CAS, Scopus and Google Scholar

- Research which is freely available for redistribution

Submit your manuscript at www.biomedcentral.com/submit 